\title{
La producción forestal en el estado de Hidalgo de 2000-2016
}

Juan José Nieto Flores, Pasante de Economía, Universidad Autónoma del Estado de Hidalgo.

Jozelin María Soto-Alarcón; Profesora Investigadora, Universidad Autónoma del Estado de Hidalgo.

Email: jmsoto@uaeh.edu.mx. ORCID_ID: 0000-0003-3931-9310

Fechas del Artículo:

Recibido: 30/08/2020

Dictamen: 06/09/2020

Aceptado:31/10/2020

Publicado: 05/11/2020
Forma de citar este artículo: Nieto J. y Soto-Alarcon J.

(2020). La producción forestal en el estado de Hidalgo de 2000-2016, Revista Científica de Estudios Urbano Regio-

nales Hatsö-Hnini, Vol 1 No. 3 pp.24-34, DOI https://

doi.org/10.47386/2020V1N3A3

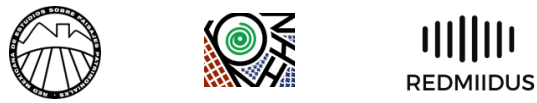

\section{Resumen}

El artículo analiza la actividad forestal en el estado de Hidalgo, México a partir del estudio del marco legal, los datos de producción y comercio, y las amenazas biológicas en el periodo de 2000 a 2016. La dasonomía es el marco referencial para identificar las condiciones de la propiedad forestal ejidal, pública y privada. Las variables analizadas son el aprovechamiento maderable, el volumen y el valor del comercio de especies maderables y no maderables, así como las amenazas fitosanitarias, con datos del Instituto Nacional de Estadística y Geografía (INEGI), Procuraduría Federal de Protección al Ambiente (PROFEPA), Comisión Nacional Forestal (CONAFOR) y Secretaría del Medio Ambiente y Recursos Naturales (SEMARNAT).

Palabras claves: Recursos naturales; ambiente; Medioambiente y comercio; Comunes

\section{Abstract}

The paper analyses the forestry activity in Hidalgo, Mexico, drawing on legal framework, production and merchandizing values, and forestry threats from 2000 to 2016 . Based on the analysis of dasotomy as theoretical framework forestry property are characterized: collective (ejidos), private and public. Timber use, volume and value of trade in timber and nontimber species; as well as phytosanitary threats are discussed, with data of the Instituto Nacional de Estadística y Geografía (INEGI), Procuraduría Federal de Protección al Ambiente (PROFEPA), Comisión Nacional Forestal (CONAFOR) and Secretaría del Medio Ambiente y Recursos Naturales (SEMARNAT).

\section{Keywords: Natural resources; Environment;}

\section{Environment and trade; Commons}

\section{Introducción}

En el estado de Hidalgo cerca del $51.55 \%$ de la superficie es forestal; de los 2,081,300 ha, que comprende la entidad (INEGI, Marco Geoestadístico 2011) 1,072,997 son superficies forestales (SEMARNAT, 2016). Las asociaciones de flora son clasificadas en superficies arboladas y otras áreas forestales (Dirección General de Gestión Forestal y de Suelos, 2002). Las superficies arboladas están conformadas por bosques y selvas.

En Hidalgo los bosques comprenden cerca de 230,743 ha, desde el noroeste hacia el sureste de la entidad, en regiones montañosas de clima templado. En su mayoría están constituidos por especies de coníferas como pino y oyamel, además de especies latifoliadas como el encino (Dirección General de Gestión Forestal y de Suelos, 2002). Las selvas (selvas altas y bosques tropicales mesófilos) abarcan 172,942 ha, estas se ubican al norte del estado en el clima cálido con lluvias regulares y al centro en una pequeña región de los municipios de Metztitlán y San Agustín Metzquititlán (Programa Estratégico Forestal para el estado de Hidalgo, 2008). Las otras áreas forestales se subdividen en zonas áridas y áreas perturbadas. Las primeras cuentan con mezquitales, huizaches, chaparrales, matorrales subtropicales y xerófilos que se extienden sobre 377,422 ha, prevalecen en el centro y sur de la entidad. Las áreas perturbadas abarcan 291,890 ha, son terrenos en los que la vegetación ha sido destruida por desmontes, incendios, agricultura o pastoreo (Programa Estratégico Forestal para el estado de Hidalgo, 2008). 


\section{La producción forestal en el Estado de Hidalgo de 2000-2016}

Juan José Nieto Flores, Jozelin María Soto-Alarcón

Las principales actividades económicas vinculadas con el aprovechamiento forestal son la producción de madera y papel, a partir del aserrado de tablas y tablones, la conservación de madera y fabricación de productos de madera para uso industrial, para la construcción, embalaje, envases, materiales, artículos y utensilios para el hogar, así como productos de cartón y papel. Dichas industrias emplean a más de 353,978 personas, $42.83 \%$ son mujeres y el $57.17 \%$ son hombres (INEGI, Censo Económico 2014).

\section{Objetivos}

La producción y valorización de las principales actividades económicas asociadas al sector forestal son analizadas a partir del marco legal y las amenazas biológicas, con la finalidad de desarrollar un panorama y generar alternativas de fomento en la entidad. En un primer momento, se explora el papel de la ley de desarrollo sustentable para fomentar la producción y rentabilidad del sector. Posteriormente se estudia la evolución de la producción maderable y no maderable y el valor de la producción de las principales especies utilizadas. Después, se exponen las principales amenazas biológicas y en las conclusiones se sugieren alternativas para hacer frente a los escenarios expuestos.

\section{Metodología}

La Estrategia Nacional de Manejo Forestal Sustentable para el Incremento de la Producción y Productividad (2013) media la interacción entre los humanos y el medio ambiente a partir de cinco dimensiones para la sustentabilidad y el aprovechamiento forestal. Las dimensiones comprenden el fortalecimiento empresarial comunitario, la silvicultura y manejo forestal (técnico-ambiental), el abasto, la transformación y los mercados, así como la coordinación institucional y el monitoreo y evaluación (CONAFOR, 2013). La noción de dasonomía se utiliza para analizar la interacción entre las variables involucradas desde un enfoque sistémico que integra la sustentabilidad biológica y económica (United States Agency of International Development, 2015). El análisis de la industria contempla el proceso de transformación de los recursos forestales en bienes de consumo e integra el concepto de sanidad vegetal para identificar las amenazas fitosanitarias del sector.

\section{Resultados}

Ley General de Desarrollo Forestal Sustentable (LGDFS) regula las actividades forestales. En el Capítulo II, artículo 15 establece las bases para la ope- ración de la CONAFOR y sus Atribuciones (LGDFS, 2018). La CONAFOR es un organismo público descentralizado encargado de desarrollar, favorecer e implementar las actividades productivas, de conservación, producción, comercialización y restauración en materia forestal.

En el estado de Hidalgo se autorizaron programas de manejo forestal para una superficie de 2,598 ha, equivalentes a un volumen de 284,518 metros cúbicos en rollo $\left(\mathrm{m}^{3} \mathrm{r}\right)$ (CONAFOR, 2016). Del total de autorizaciones 2,343 corresponden a propiedad privada y 255 a núcleos agrarios: seis comunidades y 249 son propiedad ejidal. Dichas autorizaciones produjeron anualmente 126,050 $\mathrm{m}^{3} \mathrm{r}$ (Anuario Estadístico Forestal, 2016). El $75 \%$ de las autorizaciones ejecuta el Sistema Silvícola para Bosques Regulares, apoyado con el método de ordenación "Método de Desarrollo Silvícola", el resto de las autorizaciones corresponden a otros métodos como el Método Mexicano de Ordenación de Bosques Irregulares y Saneamientos (Programa Estratégico Forestal para el estado de Hidalgo, 2008).

En el Capítulo I, artículo 7, Fracción XXXVIII de la LGDFS se establece que la ordenación forestal es la organización económica de la superficie forestal, tomando en cuenta sus características silvícolas que implican la división espacial y temporal de las actividades del manejo forestal (LGDFS, 2018). Establece que las Unidades de Manejo Forestal (UMAFORES) son los territorios con condiciones físicas, ambientales, sociales y económicas semejantes que hacen posible su ordenación, manejo forestal sustentable, producción, transformación, distribución, comercialización y conservación de los recursos (LGDFS, 2018). En el estado de Hidalgo son reconocidas cinco UMAFORES, ver mapa 1 (Programa Estratégico Forestal para el estado de Hidalgo, 2008).

A partir del 2000 se han registrado variaciones minúsculas en la superficie forestal de la entidad, manteniéndose alrededor de 1,072,997 ha, durante los 17 años analizados (SEMARNAT, Anuarios Estadísticos Forestales 2000-2016). Las principales comunidades arbóreas que producen bienes maderables están conformadas por coníferas y latifoliadas (Programa Estratégico Forestal para el estado de Hidalgo, 2008). Las coníferas que predominan en la entidad son pino, abeto, oyamel, cedro y enebro. Las latifoliadas más abundantes son árboles de hoja ancha como el encino (SEMARNAT, Compendio de Estadísticas Ambientales 2010). 
Figura 1. UMAFORES del estado de Hidalgo, 2008

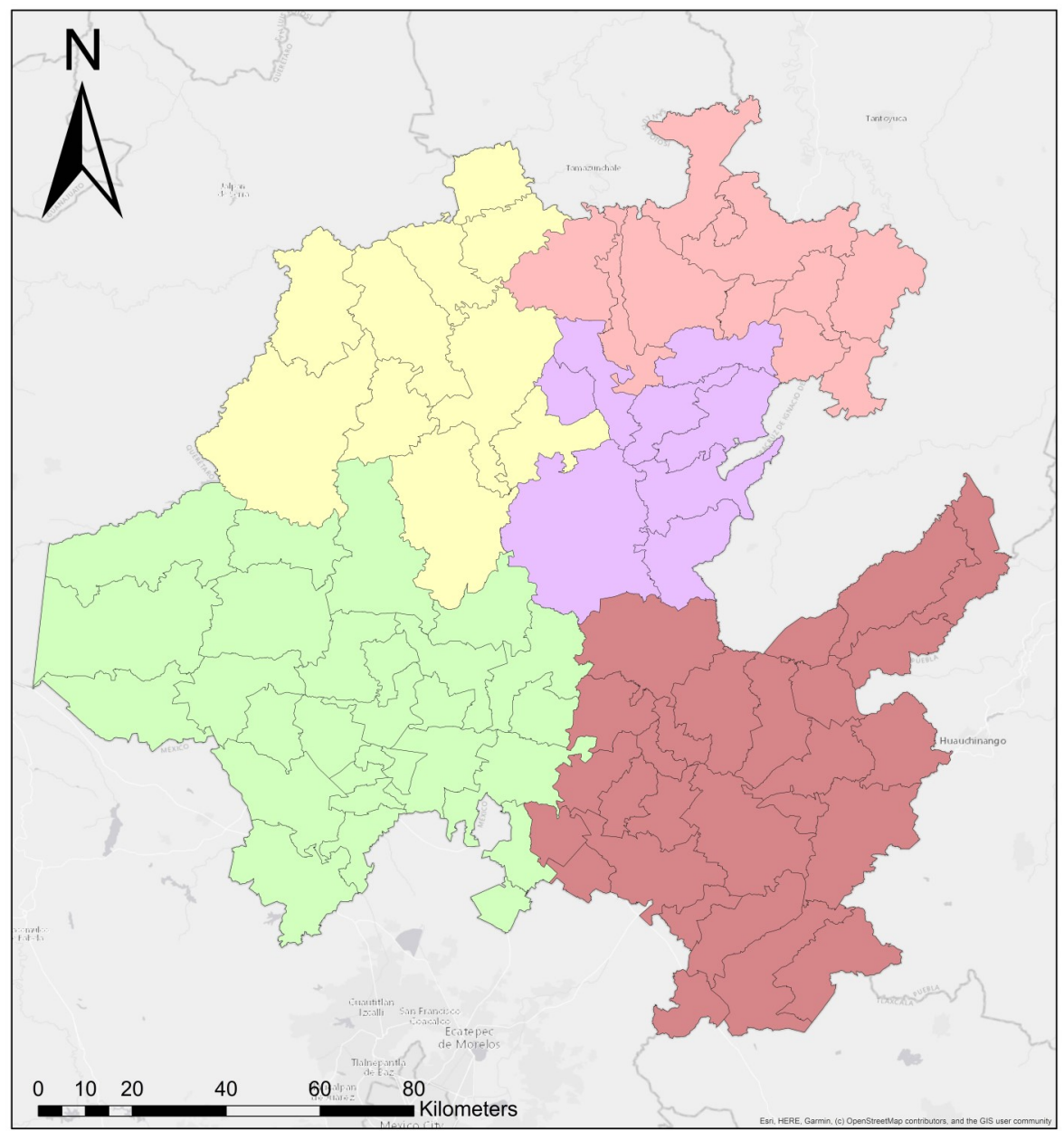

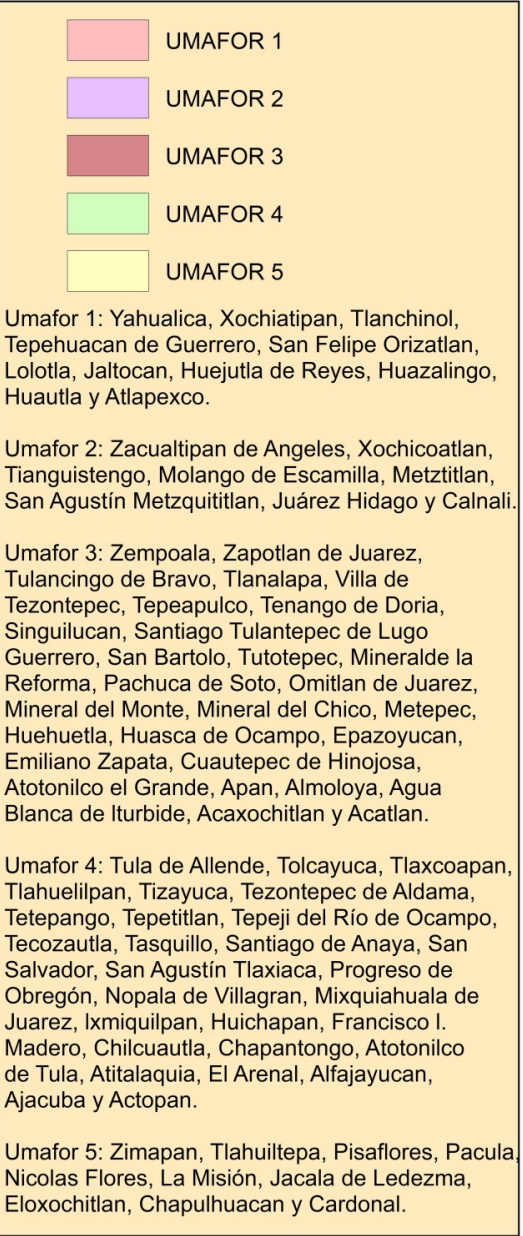

Fuente: Elaboración propia con base en el Programa Estratégico Forestal para el Estado de Hidalgo, 2008.

\section{Análisis de la producción}

Los datos recopilados en el Anuario Estadístico Forestal del año 2016, indican que el estado de Hidalgo mantenía alrededor de 1,072,997 has de superficie forestal. Con respecto a la producción maderable se contabilizaron $94245 \mathrm{~m}^{3} \mathrm{r}$ de pino, 6,397 de oyamel, 80 de otras coníferas, 23,135 de encino, 2,054 de otras latifoliadas, que en conjunto suman una producción maderable de $125,912 \mathrm{~m}^{3} \mathrm{r}$ para el año 2016 y con un valor de $122,469,020$ pesos. Todos los valores monetarios presentados son a precios corrientes de 2013 (SEMARNAT, Anuario Estadístico Forestal, 2016).

En cuanto a la producción no maderable del año 2016, se registró la producción de 21 toneladas de fibras naturales con un valor de 400,634 pesos; 94 toneladas de tierra de monte con valor de 14,103 pesos y 23 toneladas de productos catalogados como "otros" (dentro de los cuales se encuentran insectos, hierbas aromáticas, plantas medicinales, semillas, cortezas, etc.) con un valor de 92,655 pesos (SEMARNAT, Anuario Estadístico Forestal 2016).

En la gráfica 1 se observa que la producción y el precio han presentado tendencias crecientes, sin embargo, el valor de la producción ha mantenido un mayor ritmo de crecimiento. Durante los primeros seis años el valor aumentó, en 2006 la proporciónprecio fue de 1:1000, lo que significa que el valor de un metro cúbico en rollo de producción maderable rondaba los mil pesos. 
Después de este año la proporción disminuyó, pero medio anual: la de la producción es de solo 0.055 las cifras se mantuvieron cercanas, siendo hasta el \%, mientras que la de su valor es de $5.86 \%$. Esto año 2008 cuando el valor del $\mathrm{m}^{3} \mathrm{r}$ de producción ma- significa que la producción ha aumentado de forma derable sobrepasó los mil pesos. Existe una diferen- ínfima, pero el valor de esa producción ha aumentacia significativa entre las tasas de crecimiento pro- do a un mayor ritmo.

\section{Gráfica 1. Producción $\left(\mathrm{m}^{3} r\right)$ y valor de la producción maderable del estado de Hidalgo (miles de pesos a valor corriente de 2013) 2000-2016}

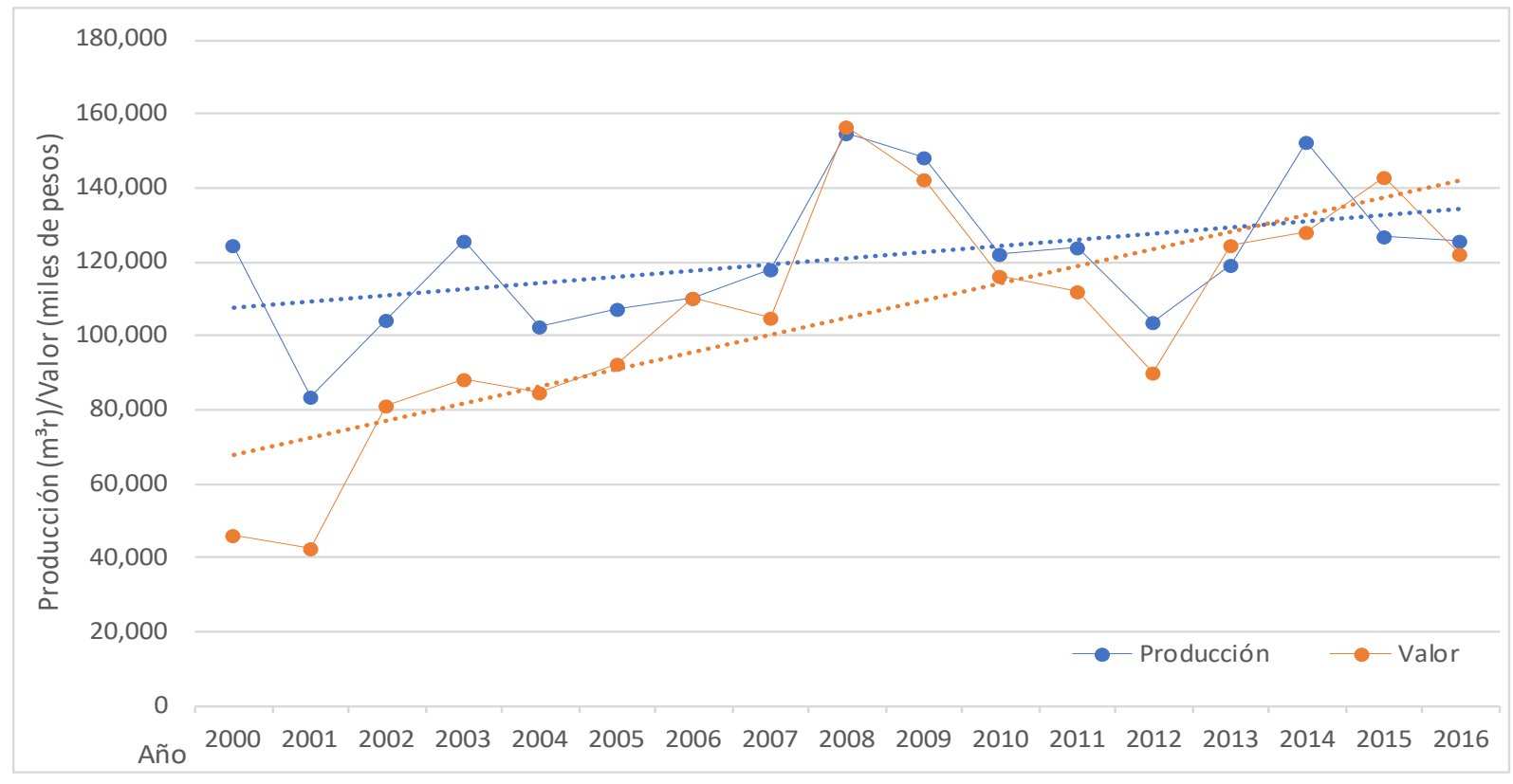

Fuente: Elaboración propia con base en SEMARNAT, Anuarios Estadísticos Forestales, 2000-2016.

Para el 2008, la producción maderable fue de $154,135 \mathrm{~m}^{3} \mathrm{r}$ con un valor de $156,784,752$ pesos (SEMARNAT, Anuario Estadístico Forestal 2008), Del año 2000 al 2016, Atitalaquia fue el municipio con mayor producción bruta. En 2014 aportó el $64.71 \%$ del total de la producción estatal, contó con 1,227 unidades económicas que emplearon a 15,276 personas en la industria forestal. (INEGI, Censo Económico 2014). En el mismo año, los municipios con mayor producción después de Atitalaquia fueron: Pachuca de Soto, Tepeji del Río de Ocampo y Tizayuca, los cuales aportaron $6.1 \%, 5.4$ $\%$ y $5.1 \%$ respectivamente y en conjunto, estos tres municipios sumaron 24,448 unidades económicas enroladas en la industria forestal, emplearon a 121,510 personas (INEGI, Censo Económico 2014). La concentración productiva en Atitalaquia se puede liada más producidas. explicar por la presencia de pequeñas y medianas empresas con una mayor capacidad productiva que en otros municipios, en promedio, las empresas en Atitalaquia operan con 13 empleados, mientras que, en los otros tres municipios con mayor producción las empresas emplean a cinco personas (INEGI, Censo Económico 2014).

En la gráfica 2 es notable que la producción de pino fue superior a la de cualquier otra especie maderable y la segunda con mayor producción fue el encino. La producción de pino y encino tienen tendencias al alza, esto sugiere que en los próximos años la producción de ambas especies aumente. Sin embargo, otras coníferas y latifoliadas han mantenido tendencias de producción decrecientes, lo que propone un escenario opuesto al de la conífera y latifo- 
Es interesante observar que, aunque sus puntos tos similares. En el estado de Hidalgo también se máximos y mínimos no coinciden, sus líneas de ten- producen especies maderables preciosas como caodencia son paralelas, lo que significa que sus pen- ba, cedro rojo y roble.

dientes son similares, manteniendo comportamien-

\section{Gráfica 2. Producción maderable del estado de Hidalgo $\left(m^{3} r\right)$ 2000-2016}

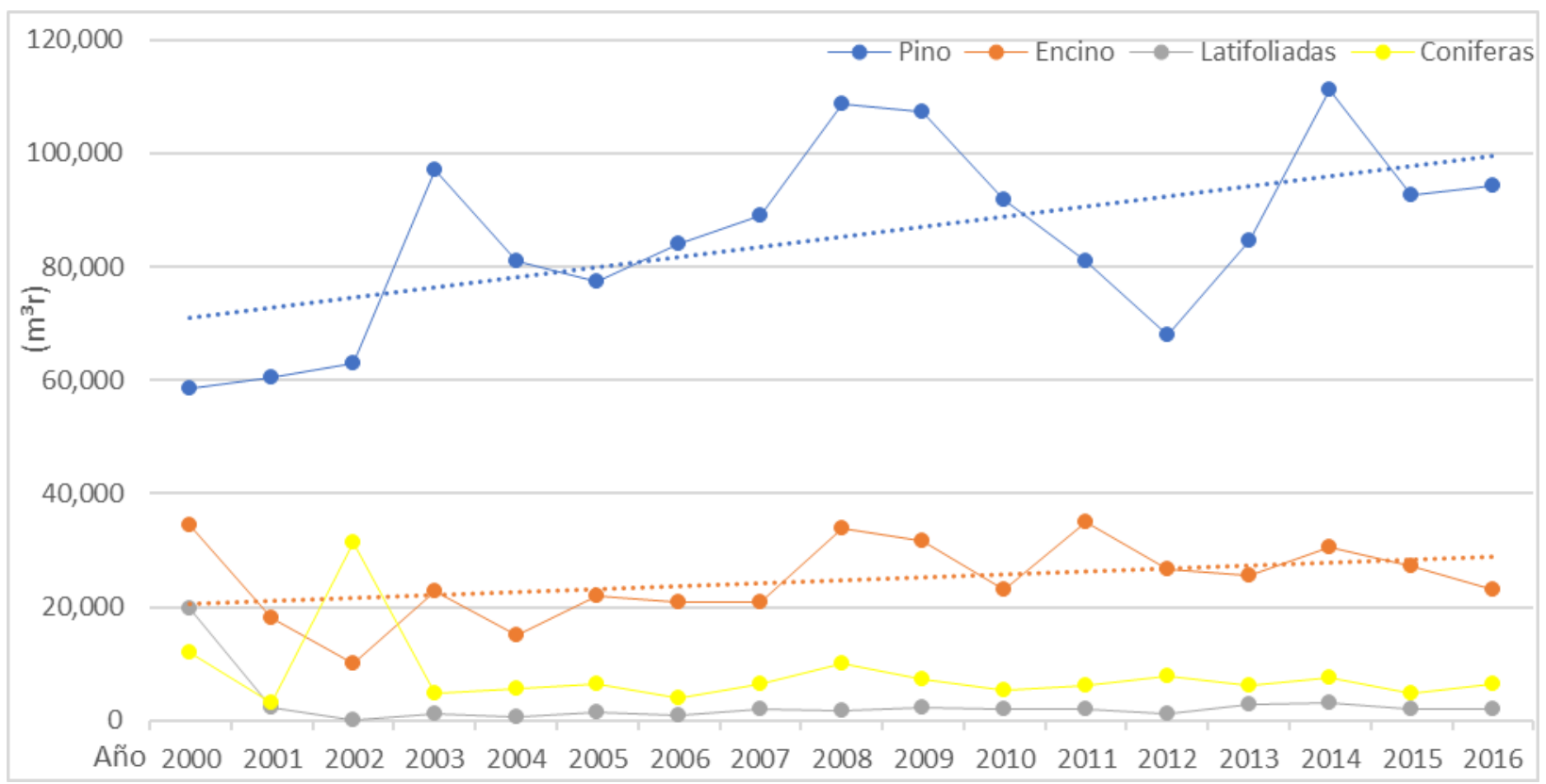

Fuente: Elaboración propia con base en SEMARNAT, Anuarios Estadísticos Forestales 2000-2016.

La producción de maderas preciosas se registró en otros productos de madera son leña, celulósicos y los años 2005, 2006, 2007, 2012, 2013 y 2014, con carbón. Los celulósicos son producidos con celulosa niveles que van de los 4 a los $380 \mathrm{~m}^{3} \mathrm{r}$ y valores que extraída de la madera, se utilizan en la fabricación van de los 3,696 a los 570,428 pesos (SEMARNAT, de papel, cartón y corchos, su producción ha preAnuarios estadísticos Forestales 2000-2016). La in- sentado puntos máximos en 2000, 2003 y 2013 , dustria de la madera hidalguense involucra las si- años en los que la producción de leña y carbón disguientes actividades económicas: Aserrado y con- minuyó (SEMARNAT, Anuarios Estadísticos Foresservación de la madera, aserrado de tablas y tablo- tales 2000-2016).

nes, fabricación de productos de madera para la construcción, para embalajes y envases, fabricación de materiales trenzables, de utensilios y artículos para el hogar, de productos industriales y otros productos de madera (INEGI, Censo Económico 2014).

Los productos no maderables del estado de Hidalgo se clasificaron en el rubro "otros". A partir del 2011 se registra la producción de fibras naturales y el siguiente año se incorpora la valoración de la tierra de monte. Desde 2013 se observa la caída en la proEn la gráfica 3 se observa el comportamiento de la ducción y llega a su punto más bajo en el 2015. Paindustria maderera hidalguense con datos de la pro- ra el 2016 ya no se produjeron semillas, gomas, reducción de madera para la construcción y la fabrica- sinas, insectos, hierbas, etc. La tasa de crecimiento ción de otros productos de madera. Los productos promedio anual de estos productos ha sido de para la construcción fabricados en Hidalgo son es- $0.72 \%$, esto debido a los altibajos sufridos a lo largo cuadría, postes, pilotes y morillos, mientras que los del periodo analizado. 
La producción forestal en el Estado de Hidalgo de 2000-2016

Juan José Nieto Flores, Jozelin María Soto-Alarcón

Gráfica 3. Producción forestal maderable anual del estado de Hidalgo 2000-2016 ( $\left.m^{3} r\right)$

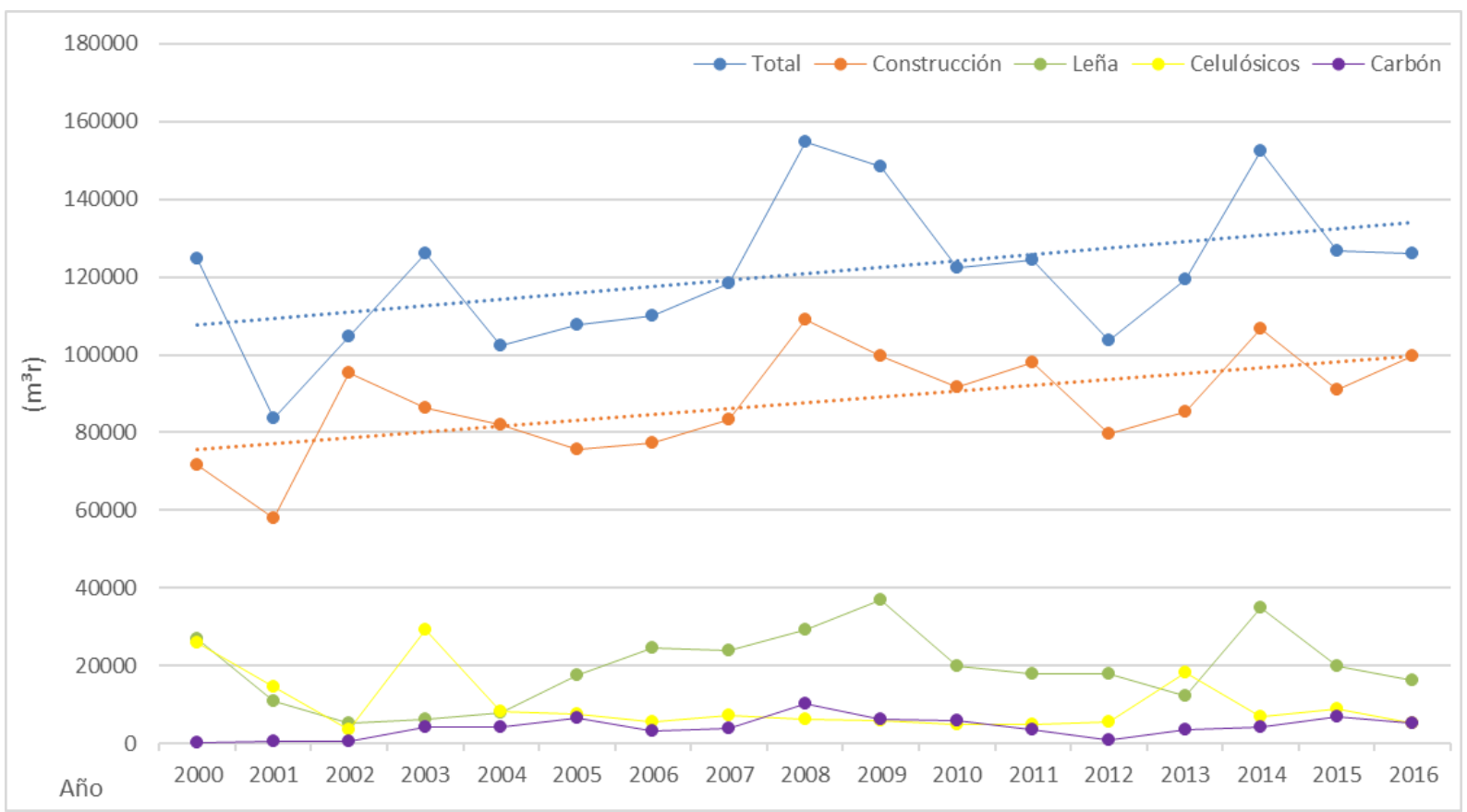

Fuente: Elaboración propia con base en SEMARNAT, Anuarios Estadísticos Forestales 2000-2016.

Gráfica 4. Producción forestal no maderable anual del estado de Hidalgo (toneladas)

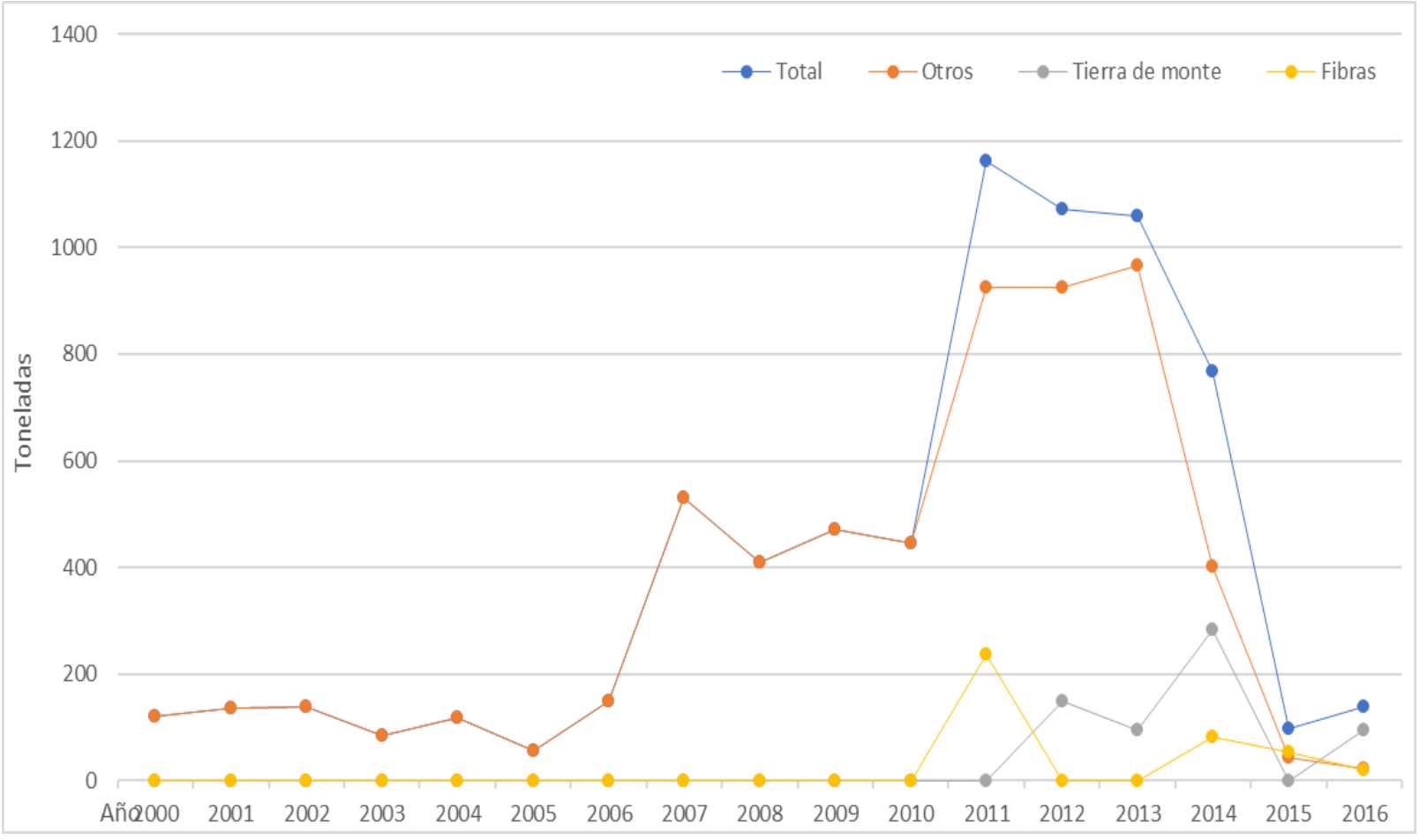

Fuente: Elaboración propia con base en SEMARNAT, Anuarios Estadísticos Forestales 2000-2016. 


\section{Análisis del valor de la producción}

Al comparar las gráficas 2 y 5 se observa que el comportamiento de las latifoliadas y de otras coníferas es sincrónico, ya que los altibajos se registran en los mismos años. Solo las gráficas que ilustran la producción y valor de la producción de pino presentan variaciones del año 2001 al 2007 ya que el incremento en el valor fue mayor que la producción, pero desde el 2008 al 2012 la caída en el valor fue más precipitada que en la producción, lo que indica una caída en los precios. Del año 2012 al 2014 la producción creció a un ritmo mayor que el valor, pero en el año 2015 la producción volvió a descender de forma brusca mientras que el valor de esa producción aumentó. La tendencia del valor es mayor que la tendencia de la producción, por lo que se podría esperar que en los siguientes años continúe apreciándose la producción de pino. En 2008 el valor de la producción de pino alcanzó su punto máximo, siendo este de 124,955,259 pesos (SEMARNAT, Anuario Estadístico Forestal 2008).

Gráfica 5. Valor de la producción maderable del estado de Hidalgo $2000-2016$ (a precios corrientes de 2013)

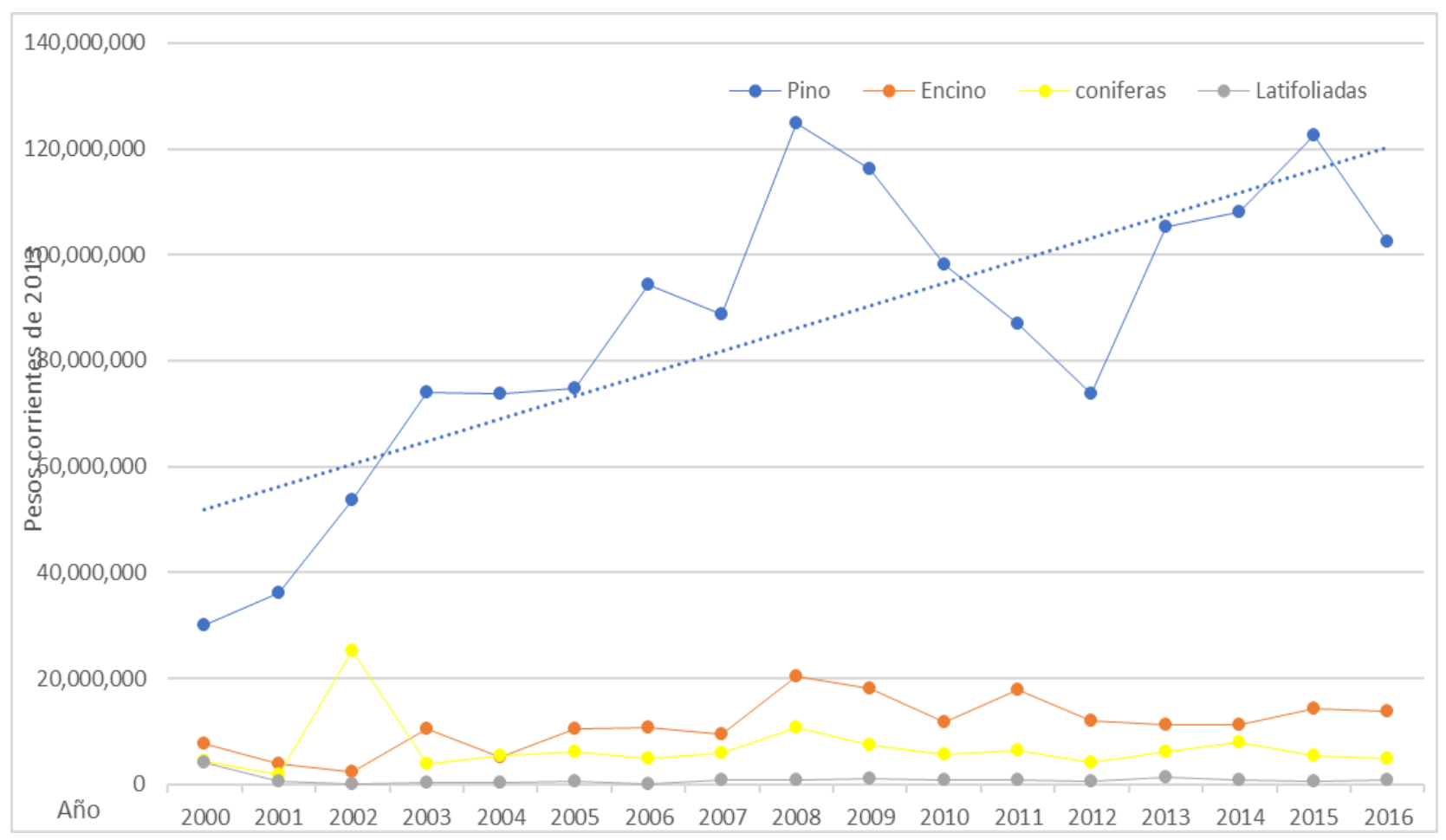

Fuente: Elaboración propia con base en SEMARNAT, Anuarios Estadísticos Forestales 2000-2016.

Los productos para la construcción son importantes 23,355,468 pesos. De dicha producción, el $52.34 \%$ para el sector, entre ellos la escuadría fue el produc- fueron otros productos no maderables, los cuales to más importante en el 2008, representó el $69.39 \%$ aportaron el $98.22 \%$ del valor (ver gráfica 6 y 7) de la producción y el $88.76 \%$ del valor total de la (SEMARNAT, Anuario Estadístico Forestal 2014). La producción. El valor de la producción del rubro pro- tasa promedio anual del valor de estos productos es ductos no maderables fue de 5,346,050 pesos de $-3.23 \%$, esto significa que a lo largo de esos (SEMARNAT, Anuarios Estadísticos Forestales años los productos no maderables han perdido valor 2000-2016). El valor máximo se registró en el 2014, en el mercado.

se produjeron 768 toneladas con un valor de 
La producción forestal en el Estado de Hidalgo de 2000-2016

Juan José Nieto Flores, Jozelin María Soto-Alarcón

Gráfica 6. Valor de la producción forestal maderable anual del estado de Hidalgo 2000-2016 (a precios corrientes de 2013)

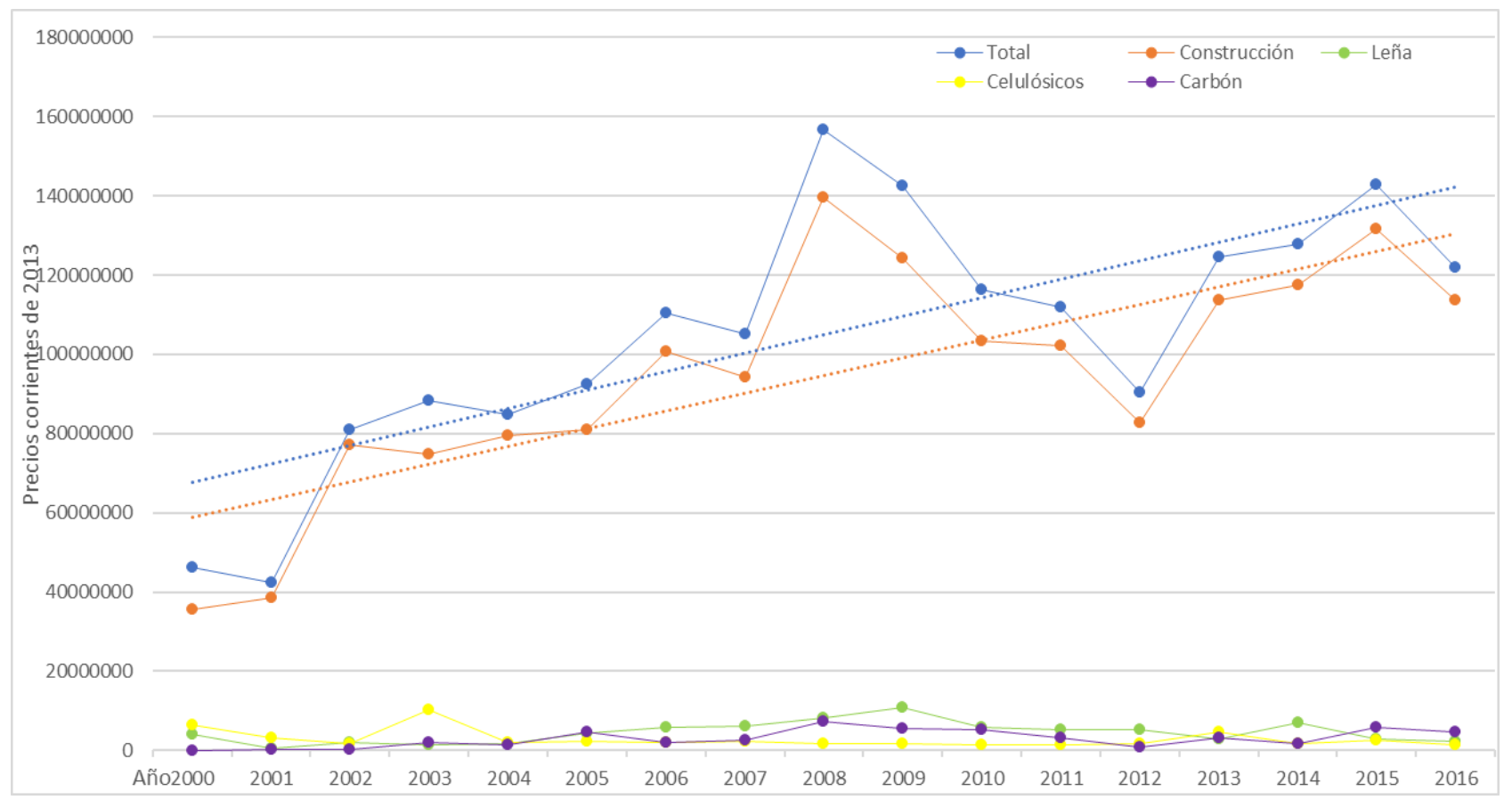

Fuente: Elaboración propia con base en SEMARNAT, Anuarios Estadísticos Forestales 2000-2016.

Gráfica 7. Valor de la producción forestal no maderable anual del estado de Hidalgo (pesos corrientes de 2013)

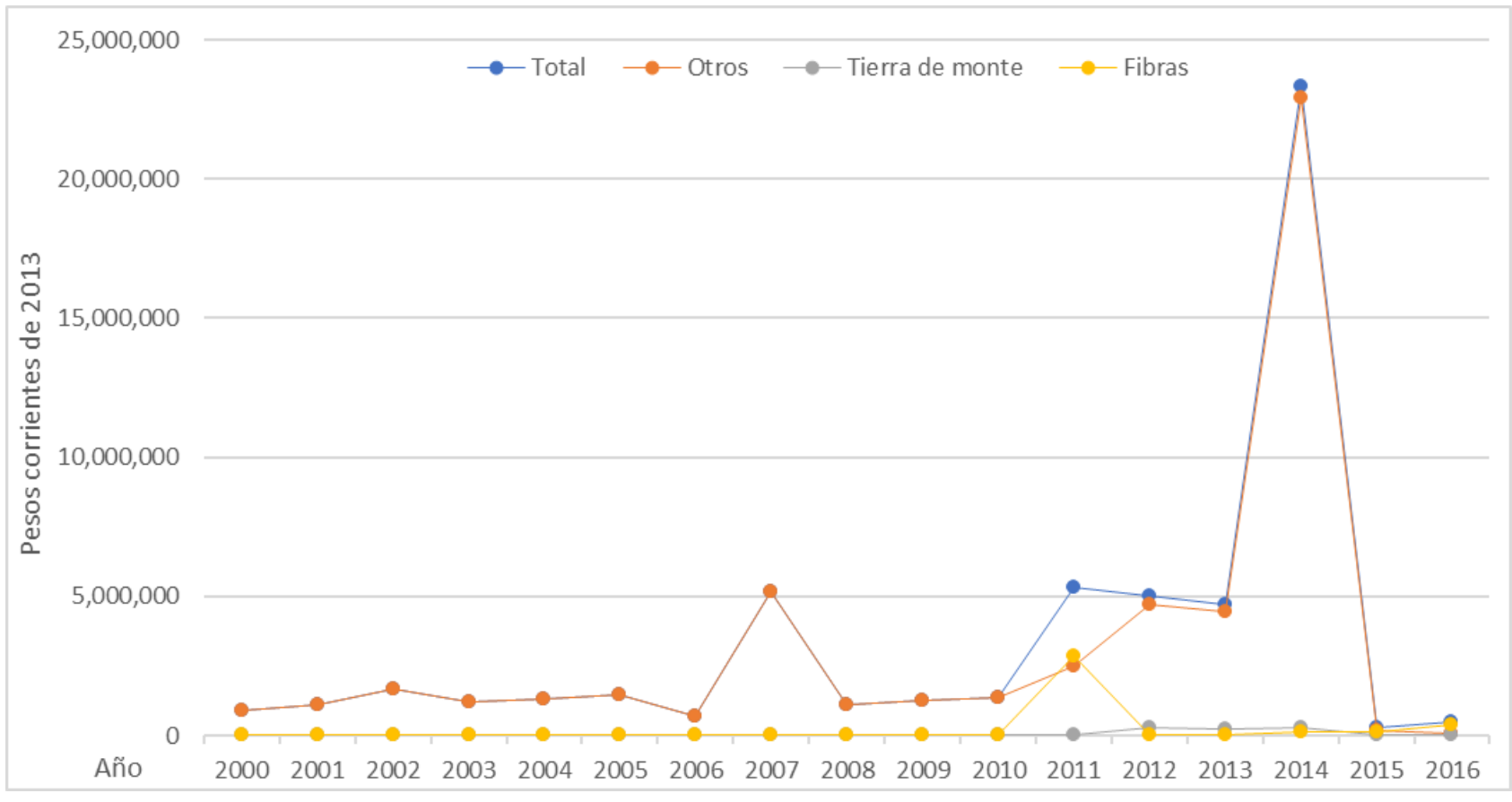

Fuente: Elaboración propia con base en SEMARNAT, Anuarios Estadísticos Forestales 2000-2016. 
Amenazas fitosanitarias en el estado de Hidalgo que las hojas se tornen amarillentas y caigan 2000-2016 (CONAFOR, 2016).

Las amenazas fitosanitarias como insectos descor- Para el año 2000 se diagnosticaron 260,000 casos tezadores y muérdago impactan la sustentabilidad de insectos descortezadores, la cantidad de casos ambiental y económica del sector, ya que aumentan disminuyeron hasta el 2007. En 2015 se notificaron la precariedad de las áreas arboladas y reduce la 119 casos cuya merma equivale a un volumen de capacidad para aportar servicios y productos fores- 217,399 $\mathrm{m}^{3} \mathrm{r}$ (SEMARNAT, Anuarios Estadísticos tales (CONAFOR, 2016). El muérdago es una planta Forestales 2000-2016). Del 2000 al 2016 se diagparasitaria que se nutre de los árboles, provocando nosticaron casos de esta plaga, particularmente en que estos no consigan los nutrientes necesarios pa- el año 2005 y 2006 la plaga afectó un área de 107 ra sobrevivir. Los insectos descortezadores se ali- metros cuadrados, pero no se registró ningún tratamentan del tronco de los árboles, dificultando así la miento para los árboles afectados (SEMARNAT, transportación de alimento a las ramas, provocando Anuarios Estadísticos Forestales 2000-2016).

Tabla 1. Amenazas fitosanitarias en el estado de Hidalgo 2000-2016

\begin{tabular}{lllllllll}
\hline \multirow{2}{*}{ Año } & \multirow{2}{*}{$\begin{array}{c}\text { Numero de } \\
\text { notificaciones }\end{array}$} & \multicolumn{2}{c}{ Descortezador } & \multicolumn{3}{c}{ Muérdago } & \multicolumn{3}{c}{ Total } \\
2016 & 35 & nd & Superficie & Volumen & Superficie & Volumen & Superficie \\
2015 & 119 & nd & nd & nd & nd & 3,107 & 1,515 \\
2014 & 53 & nd & nd & nd & nd & 217,399 & 2,021 \\
2013 & 41 & 682 & nd & nd & 14,750 & 1,619 \\
2012 & 44 & nd & nd & nd & nd & 3,603 & 3,169 \\
2011 & 31 & 1,885 & 601 & nd & 610 & 1,885 & 1,211 \\
2010 & 34 & 1,863 & 233 & nd & 1,394 & 1,863 & 1,627 \\
2009 & 66 & 5,726 & 749 & 416 & 794 & 6,142 & 1,543 \\
2008 & 43 & 9,962 & 2,094 & 615 & nd & 9,962 & 2,709 \\
2007 & 16,061 & 959 & 959 & 0 & 0 & 959 & 959 \\
2006 & $5,349.6$ & $1,557.2$ & $1,557.2$ & 107 & 0 & $1,664.2$ & $1,557.2$ \\
2005 & 12,020 & 467 & 467 & 34 & 34 & 501 & 501 \\
2004 & $30,183.7$ & 1,090 & 1,090 & 0 & 0 & 1,090 & 1,090 \\
2003 & 353 & 14 & 10 & 0 & 0 & 14 & 10 \\
2002 & 80,000 & 305 & 47 & 0 & 0 & 305 & 47 \\
2001 & 49,500 & 136 & 110 & 0 & 0 & 136 & 110 \\
2000 & 260,000 & 374 & 327 & 0 & 0 & 374 & 327 \\
\hline
\end{tabular}

Fuente: elaboración propia con base en SEMARNAT, Anuarios Estadísticos Forestales 2000-2016. 


\section{La producción forestal en el Estado de Hidalgo de 2000-2016}

Juan José Nieto Flores, Jozelin María Soto-Alarcón

\section{Conclusiones}

Hidalgo cuenta con una importante masa forestal que cubre la mitad de la extensión territorial del estado, dispone de bosques de latifoliadas, coníferas, mesófilos, selvas altas y vegetación propia de zonas áridas como mezquitales y huizaches. Estas superficies forestales están protegidas por la Ley General de Desarrollo Forestal Sustentable, la cual las ordena en Unidades de Manejo Forestal para buscar una mejor organización productiva y de mantenimiento. En la entidad se otorgan autorizaciones de explotación forestal, los cuales garantizan el manejo forestal sustentable del estado. Dichas autorizaciones deben cumplir con diferentes métodos, como son el Método de Ordenación, el Método de Desarrollo Silvícola y el Método Mexicano de Ordenación de Bosques irregulares y Saneamientos.

En el periodo 2000-2016, el estado de Hidalgo produjo bienes maderables y no maderables con mayor valor: pino, encino, escuadría y otros productos no maderables. Sin embargo, la mayor parte de la producción bruta está concentrada en un solo municipio. Los cuatro municipios con mayor producción aportan el $81.31 \%$ del total y pertenecen a las UMAFOR 3 y 4, localizadas al sur del estado. La industria maderera y la producción de papel aportaron poco más del $1 \%$ del producto interno bruto de Hidalgo (INEGI, 2014) y aunque el estado ha implementado programas federales y estatales para que las unidades económicas dedicadas a la producción de bienes maderables obtengan apoyos financieros.

Aunque se han registrado amenazas fitosanitarias en pequeña escala, es necesario prevenir y atender los casos, pues podrían afectar las capacidades productivas de todos los bienes forestales. Si bien, la producción maderable en Hidalgo ha aumentado, solo lo ha hecho con base en dos especies de árbol, el pino y el encino, y el valor de esa producción ha aumentado a un ritmo más acelerado. El escenario que presenta la producción de otros tipos de árboles es más limitado, tanto la cantidad producida como el valor de esta presentaron tasas decrecientes y afecta a la sustentabilidad ya que sembrar una especia limita la biodiversidad. Esta producción se concentra en cuatro municipios con unidades económicas que operan a pequeña escala, en el resto de municipios solo operan pequeños productores.

\section{Bibliografía}

Comisión Nacional Forestal. (2016). En busca de los descortezadores. Ciudad de México, México.

Comisión Nacional Forestal. (2013). Estrategia Nacional de Manejo Forestal Sustentable para el Incremento de la Producción y Productividad 2013-2018. Jalisco, México.

Diario Oficial de la Federación. (2018). Ley General de Desarrollo Forestal Sustentable. Ciudad de México, México.

Dirección General de Gestión Forestal y de Suelos, Secretaría de Medio Ambiente y Recursos Naturales. (2002). Ciudad de México, México.

Instituto Nacional de Estadística y Geografía. (2011). Marco Geoestadístico. Ciudad de México, México.

Instituto Nacional de Estadística y Geografía. (2014). Cuéntame INEGI, Censo Económico 2014. Ciudad de México, México. Consultado el 10 de junio de 2019.

Programa Estratégico Forestal para el estado de Hidalgo. (2008). Informe de resultados 20002008. Hidalgo, México.

Secretaría de Medio Ambiente y Recursos Naturales. (2016). Anuario Estadístico Forestal. Ciudad de México, México.

Secretaría de Medio Ambiente y Recursos Naturales. (2015). Anuario Estadístico Forestal. Ciudad de México, México.

Secretaría de Medio Ambiente y Recursos Naturales. (2014). Anuario Estadístico Forestal. Ciudad de México, México.

Secretaría de Medio Ambiente y Recursos Naturales. (2013). Anuario Estadístico Forestal. Ciudad de México, México.

Secretaría de Medio Ambiente y Recursos Naturales. (2012). Anuario Estadístico Forestal. Ciudad de México, México.

Secretaría de Medio Ambiente y Recursos Naturales. (2011). Anuario Estadístico Forestal. Ciudad de México, México.

Secretaría de Medio Ambiente y Recursos Naturales. (2010). Anuario Estadístico Forestal. Ciudad de México, México. 
Secretaría de Medio Ambiente y Recursos Naturales. (2009). Anuario Estadístico Forestal. Ciudad de México, México.

Secretaría de Medio Ambiente y Recursos Naturales. (2008). Anuario Estadístico Forestal. Ciudad de México, México.

Secretaría de Medio Ambiente y Recursos Naturales. (2007). Anuario Estadístico Forestal. Ciudad de México, México.

Secretaría de Medio Ambiente y Recursos Naturales. (2006). Anuario Estadístico Forestal. Ciudad de México, México.

Secretaría de Medio Ambiente y Recursos Naturales. (2005). Anuario Estadístico Forestal. Ciudad de México, México.

Secretaría de Medio Ambiente y Recursos Naturales. (2004). Anuario Estadístico Forestal. Ciudad de México, México.

Secretaría de Medio Ambiente y Recursos Naturales. (2003). Anuario Estadístico Forestal. Ciudad de México, México.

Secretaría de Medio Ambiente y Recursos Naturales. (2002). Anuario Estadístico Forestal. Ciudad de México, México.

Secretaría de Medio Ambiente y Recursos Naturales. (2001). Anuario Estadístico Forestal. Ciudad de México, México.

Secretaría de Medio Ambiente y Recursos Naturales. (2000). Anuario Estadístico Forestal. Ciudad de México, México.

United States Agency of International Development, The Cadmus Group, Inc. (2015). Programa de Apoyo a la Gestión Ambiental Global bajo el Contrato Número GS-10F-0105J, Pauta Ambiental Sectorial, Dasonomía. Estados Unidos. 